\title{
Computational design of drug candidates for influenza A virus subtype H1N1 by inhibiting the viral neuraminidase-1 enzyme
}

\author{
USMAN SUMO FRIEND TAMBUNAN* \\ ARLI ADITYA PARIKESIT \\ YONANIKO DEPHINTO \\ FEIMMY RUTH PRATIWI SIPAHUTAR \\ Department of Chemistry \\ Faculty of Mathematics and Sciences \\ Depok, University of Indonesia
}

It is critical to seek potential alternative treatments for H1N1 infections by inhibiting neuraminidase-1 enzyme. One of the viable options for inhibiting the activity of neuraminidase- 1 is peptide drug design. In order to increase peptide stability, cyclization is necessary to prevent its digestion by protease enzyme. Cyclization of peptide ligands by formation of disulfide bridges is preferable for designing inhibitors of neuraminidase- 1 because of their high activity and specificity. Here we designed ligands by using molecular docking, drug scan and dynamics computational methods. Based on our docking results, short polypeptides of cystein-arginine-methionine-tyrosine-proline-cysteine (CRMYPC) and cysteine-arginine-aspargine-phenylalanine-proline-cysteine (CRNFPC) have good residual interactions with the target and the binding energy $\Delta G_{\text {binding }}$ of -31.7402 and $-31.0144 \mathrm{kcal} \mathrm{mol}^{-1}$, respectively. These values are much lower than those of the standards, and it means that both ligands are more accessible to ligand-receptor binding. Based on drug scan results, both of these ligands are neither mutagenic nor carcinogenic. They also show good oral bioavailability. Moreover, both ligands show relatively stable molecular dynamics progression of RMSD vs. time plot. However, based on our metods, the CRMYPC ligand has sufficient hydrogen bonding interactions with residues of the active side of neuraminidase- 1 and can be therefore proposed as a potential inhibitor of neuraminidase-1.

Keywords: H1N1, neuraminidase-1, cyclic peptide disulfide, molecular docking, molecular dynamics

Influenza A virus subtype H1N1 causes severe infections in human respiratory system. This can lead to increased risk of mortality. Neuraminidase-1 has an important role

\footnotetext{
* Correspondence; e-mail: usman@ui.ac.id
} 
U. S. F. Tambunan et al.: Computational design of drug candidates for influenza A virus subtype H1N1 by inhibiting the viral neuraminidase-1 enzyme, Acta Pharm. 64 (2014) 157-172.

in H1N1 viral replication and acts as a major target in drug design and is one of the serotypes of neuraminidase enzyme. However, H1N1 virus is evolving rapidly and some drug resistance strains are emerging. H1N1 virus is a subtype of the influenza virus A, which has become pandemic and allows the formation of a new strain that spreads from human to human (1).

Neuraminidase has the cardinal role in viral infection, especially in its virulence. Adaptability of influenza A virus in human populations was caused by biochemical changes in the ability of the cell membrane attachment from $\alpha-2,3$ sialic acid Gal to $\alpha-2,6$ sialic acid Gal receptor (2). Influenza A virus is currently able to infect humans because $\alpha-2,6$ sialic acid Gal receptor is known as a binding agent. Influenza A virus in the infected host is associated with hemagglutinin protein as an adhesive agent of the virus for gaining entrance into the host cell and neuraminidase as an agent of the virus released from the infected host cell (3).

The important role of neuraminidase was mainly in viral replication and high conservation at the active site. This makes neuraminidase a key target in the design of antiviral influenza virus agents (4). Zanamivir and oseltamivir are antiviral drugs that can inhibit neuraminidase processes (5). However, resistance to the antiviral oseltamivir has increased to $98.5 \%$ in the USA (6). To this end, it is necessary to develop a novel drug candidate that would provide better efficacy than oseltamivir.

The design and utilization of peptides are the most promising fields in the development of novel drugs due to their high activity and specificity, low toxicity and the fact that they do not accumulate in the body (7-9). Almost 200 peptides are used as drugs and approximately 400 peptides are in the preclinical phase, with an average growth of almost $20 \%$ for one year (10). These facts have made the peptide-based design for H1N1 drugs a viable alternative.

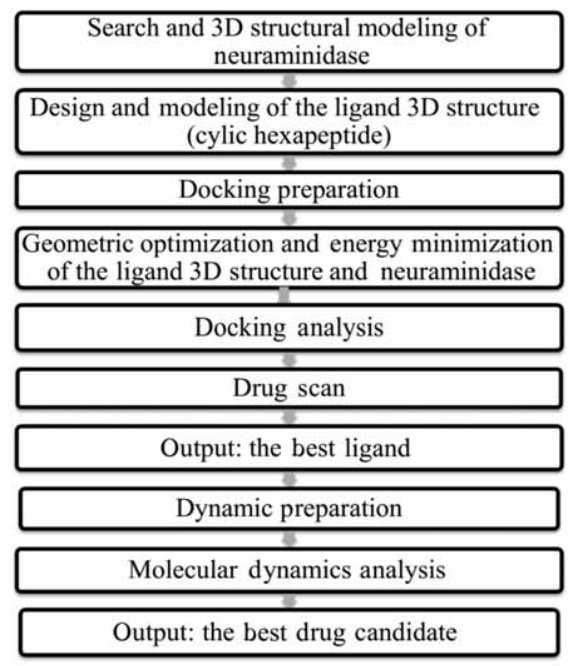

Fig. 1. The pipeline of our method. 
U. S. F. Tambunan et al.: Computational design of drug candidates for influenza A virus subtype H1N1 by inhibiting the viral neuraminidase-1 enzyme, Acta Pharm. 64 (2014) 157-172.

However, drug design should follow Lipinski's rule of five in order to obtain a robust lead compound (11). According to Lipinski's rule of five, a good drug mass should have a molecular weight of about 500, while designed hexapeptide should have a molecular weight of 700 (12). This means that the drug candidate has a molecular mass beyond the designated threshold. Although it did not pass Lipinski's rule, some peptides have been successfully utilized as drugs. For instance, the cyclic peptide cRGD (arginine-glycine-aspartic acid) has been used as a therapeutic for hepatic fibrosis (13). White et al. (14) reported that the cyclic hexapeptide of cLLLLPY (leucine-leucine-leucine-leucine-proline-tyrosine) has a molecular mass of 755 with three N-methyl groups; and showed $28 \%$ oral bioavailability in rats, as the most permeable among the tested compounds.

Nowadays, peptide drug design has been assisted by the development of computational chemistry. Hence, it is important to note that computational chemistry has played an important role in drug design with validation in wet laboratories. Docking methods used in computational drug design can be combined with molecular dynamics simulations to predict the existence of the ligand-enzyme complex. The dynamics method will eventually give a more complete description of the biochemical reaction, by exposing its energy state against the function of time. Antibiotic resistance is a serious problem nowadays, and computation of DNA gyrase enzyme and its ligands has been proven in wet labs to be correctly predicted (15). Therefore, such experiments provide strong evidence that eventually bioinformatics tools could indeed be useful for assisting wet laboratory experiments.

This study focuses on in silico design of cyclic hexapeptide, which is expected to inhibit neuraminidase-1 of influenza A virus subtype H1N1. Our method combines computational chemistry, computational biology and materials science in studying molecular systems from small chemical systems to large biological molecules (Figure 1) (16). Research was carried out using molecular docking, drug scan and molecular dynamics.

\section{EXPERIMENTAL}

We started the experiment by searching and modeling the 3D structure of neuraminidase-1, because a correct 3D representation of the structure is necessary for good biochemical interaction modeling. The ligands were designed as combinations of six amino acids (cyclic hexapeptide) (the pattern is available upon request as supplementary material). The docking procedure that successfully generated data validation for oseltamivir and zanamivir was employed in this experiment (11). Docking was done to fit the format file for molecular operating environment (MOE) and then geometric optimization and energy minimization were applied to have the best docking structural conformation. Docking analysis was done to obtain the most thermodynamically favorable ligand-protein interaction. Drug scan was conducted to observe whether the ligand was suitable to act as a drug or not. We obtained ligands that could be scaled as drug candidates. Hence, the drug candidate interaction with neuraminidase- 1 must be tested based on the durability and kinetics of the reaction. At the end, molecular dynamics analysis was conducted to obtain the most kinetically stable ligand-protein interaction. Details of our methodology are explained in the next subsections. 
U. S. F. Tambunan et al.: Computational design of drug candidates for influenza A virus subtype H1N1 by inhibiting the viral neuraminidase-1 enzyme, Acta Pharm. 64 (2014) 157-172.

\section{Neuraminidase-1 sequence retrieval}

Neuraminidase-1 protein sequence data with Genbank code ACT79135.1 were obtained as a target enzyme for the determination of the 3D structure of H1N1 neuraminidase-1. Neuraminidase 3ti4B Swiss model (PDB code) was obtained as a template that had 99.23\% similarity to neuraminidase-1 \{Influenza A virus [A/Japan/1070/2009 (H1N1)]\}.

\section{Neuraminidase-1 and ligand preparation}

Influenza H1N1 neuraminidase-1 sequences used in this study were obtained from the Natural Center for Biotechnology Information (NCBI) database of 2009-2011 (17, 18). Full neuraminidase (NA) sequences were used to design the homology structure of the online Swiss model with downloaded fast-all (FASTA) files from NCBI.

Determination of amino acid sequences was done by sequentially assigning the hexapeptides. Amino acid sequences of cyclic peptides could be designed and modeled into 3D structures. The designing and modeling procedure was done using the software of ACD labs (Advanced Chemistry Development Inc, Canada). Then, the ACD storage format was converted into cyclic peptides using Vega zz software (Department of Pharmaceutical Science, University of Milan, Milan, Italy) in order to facilitate the docking process.

\section{Molecular docking}

Docking file preparation was done by optimizing the geometry, minimizing the energy of the ligand and 3D visualization of neuraminidase-1, using MOE 2008.10 software (19). Docking process was conducted with MOE 2008.10 software. The placement method used was the triangle matcher with 1000 rounds. Scoring function used was the London $\mathrm{dG}$ displaying the best data in 100 iterations. Furthermore, out of the best data, 100 measurements were repeated using the force field refinement loop configuration with a population size of 1000 in accordance with the MOE default. From the displayed results of the whole process, the selected data were the best ones.

\section{Drug scan}

Drug scan was performed on the drug candidates to determine whether the compounds complied with Lipinski's rule of five or not (20). Some software packages such as ToxTree (Ideaconsult Ltd, Bulgaria), Osiris Property Explore (Actelion Pharmaceuticals Ltd, Switzerland) and Free ADME-Tox Filtering tools-drug (FAF-drugs) (INSERM, France) were used. Toxtree was used to compute the mutagenicity and carcinogenicity properties based on compilations of stuctural alerts (21). Osiris was used to compute the pharmacological predictions (mutagenicity, carcinogenicity, irritation, reproductive effectiveness) and drug properties ( $\log P$, solubility, drug likeness, drug score) (22). Then, FAF Drugs 2 was used to compute Lipinski's physical property $\left(M_{r}, \mathrm{HBD}, \mathrm{HBA}\right.$, and log $P$ ) and oral bioavailability based on Veber and Egan rules (23). 
U. S. F. Tambunan et al.: Computational design of drug candidates for influenza A virus subtype H1N1 by inhibiting the viral neuraminidase-1 enzyme, Acta Pharm. 64 (2014) 157-172.

\section{Molecular dynamics simulation}

Partial geometry optimization of the ligand-enzyme complex charge was done by the current force field method, which is a 'fixed-charge' model for atomic charge value representation that is kept stable under the influence of electrostatic fluctuation (24). Energy minimization was then performed with a force field MMFF94x (Merck Molecular Force Field 94x Merck Ltd., Whitehouse Station, NJ, USA). Root mean square (RMS) is a measure of the size of distribution given to electronic gradient during minimization. Zero gradient is the optimized energy. The utilized solvation is born with RMS of 0.05 gradient kcal mol A. Default parameters of the dynamics simulation were employed and the simulation output files are in MOE (Chemical Computing Group, Canada) format for dynamics simulation (25).

Molecular dynamics simulation of the ligand inhibitor was performed by MOE2008.10 MOE-dynamics. The parameters were used in accordance with the MOE-default for the dynamics NVT ensemble ( $N$ - number of atoms; $V$ - volume, $T$ - temperature) with the NPA (Nosé-Poincaré-Andersen) algorithm (26). Timing of conformational stability of the enzyme complex to solvents was performed for 100 ps at the equilibration stage. Simulations were performed at 5000 ps, temperatures of $310 \mathrm{~K}$ (the conditions of normal human body temperature) and $312 \mathrm{~K}$ (the temperature conditions of fever in humans).

\section{RESULTS AND DISCUSSION}

Although MOE 2008.10 is a reliable and cost-effective program, there is one caveat that should be considered. Docking involving standard stochastic search conditions was not efficient and applicable for the high-throughput mode (27). From the modeling results, Qualitative model energy analysis 4 (QMEAN4) score of 0.66 was obtained. This value is still within the allowable range for the reliability of models, estimated between $0-1$.

To determine the quality of the modeled structure, it was analyzed using the Ramachandran plot (28) (Fig. 2). This infers that there are no residues at all in the disallowed region. Results of the structural analysis of neuraminidase- 1 show that there is a single amino acid residue in the disallowed region or approximately $0.3 \%$ of total residues. When more than $15 \%$ of non-glycine residues are located in disallowed regions, it can be concluded that the structure of the proteins has very bad quality (very unstable), so it can be inferred that the structure of the model is of good quality and can be used for further analysis.

The information on amino acid residues in the neuraminidase- 1 active site can be seen in NCBI data. H1N1 neuraminidase-1 ACT79135.1 has seven functional amino acids that play a role in active sites. They are Arg118, Asp151, Glu278, Arg293, Arg368, Tyr402 and Glu425.

Elucidation of the amino acid sequences of peptides inhibitors is based on the properties of the amino acid residues of the H1N1 neuraminidase-1 (11). The neuraminidase- 1 enzyme has four amino acid residues that play a role in the active site, which has 
U. S. F. Tambunan et al.: Computational design of drug candidates for influenza A virus subtype H1N1 by inhibiting the viral neuraminidase-1 enzyme, Acta Pharm. 64 (2014) 157-172.

Fig. 2. Ramacandran plot of the modeled neuraminidase structure. The $\phi$ and $\Psi$ angels in the polypeptides, shown in the plot refer to the polypeptide backbone rotations in the bond between $\mathrm{N}-\mathrm{C} \alpha(\phi)$ and $\mathrm{C} \alpha-\mathrm{C}(\Psi)$.
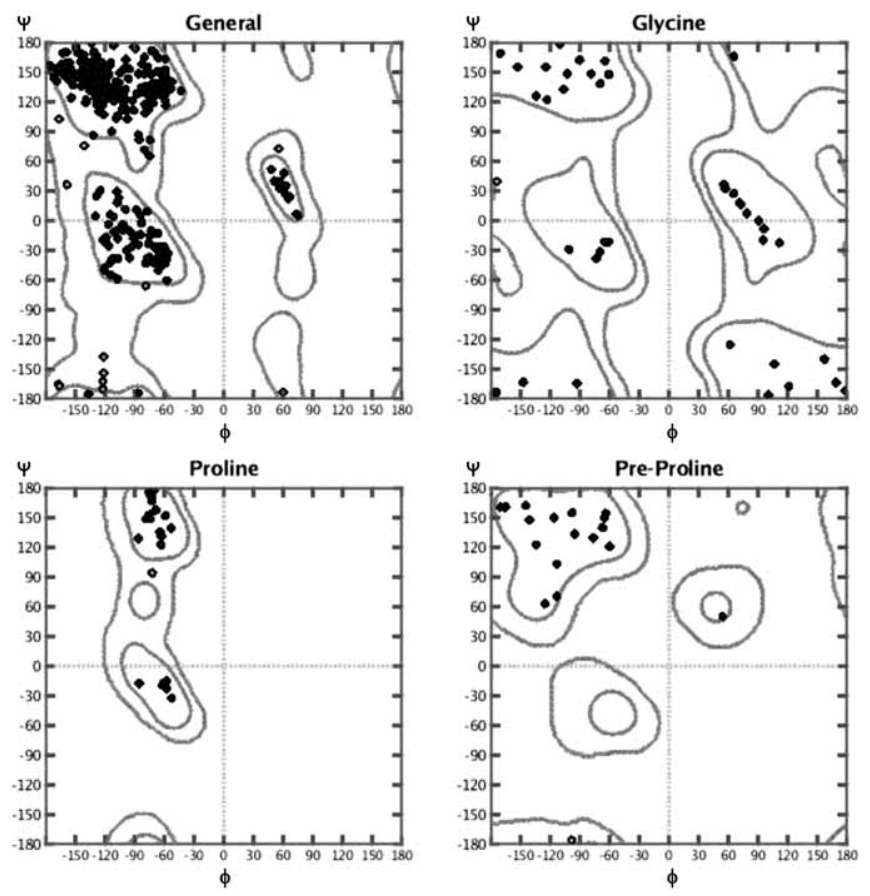

positive and negative properties of polar charge. Neuraminidase- 1 binding sites exist around Glu119, Arg152, Arg156, Trp179, Ser180, Ile223, Arg225, Glu228, Ser247, Tyr274, Glu277 and Asn295. They are polar and non-polar residues. Therefore, the cyclic peptide ligand was formed by combining four amino acids with polar and non-polar groups, and on their edges there were two cystein residues that formed a bond connecting them.

The combination of 11 polar amino acid residues (aspartic acid, asparagine, serine, trionin, glutamine, glutamic acid, lysine, histidine, tyrosine, cysteine and arginine) and nine non-polar amino acid residues (alanine, glycine, valine, leucine, isoleucine, tripthopan, phenylalanine, methionine and proline) produced 5096 cyclic hexapeptide ligands. The utilized standard ligands were oseltamivir, zanamivir, AD3BF2D and NNY.

File preparation and docking were done with energy minimization to optimize the geometry of 3D structure of neuraminidase-ligands and it was expected to eliminate undesirable interactions in the structure (bad contact) of protein structure (19). Thus, energy minimization was necessary to fix conformational problems associated with X-ray or NMR-based structures.

Molecular docking was designed to search for the proper conformation of the bond between ligand and receptor. The process was carried out on 5096 ligands and four standard ligands (zanamivir, oseltamivir, AD3BF2D, and NNY). In molecular docking, the enzyme was made rigid and the ligand on the left was flexible, so that it could freely move and rotate. The docking based-screening was performed three times, and the first screening resulted in 200 ligands that had the lowest binding free energy value. It was 
U. S. F. Tambunan et al.: Computational design of drug candidates for influenza A virus subtype H1N1 by inhibiting the viral neuraminidase-1 enzyme, Acta Pharm. 64 (2014) 157-172.

Table I. Free binding energy values $(\Delta \mathrm{G})$ of molecular docking results

\begin{tabular}{|c|c|c|}
\hline Ligand & $\Delta G\left(\mathrm{kcal} \mathrm{mol}^{-1}\right)$ & Structure \\
\hline CRMYPC & -31.7402 & \\
\hline CWEVCC & -31.5652 & \\
\hline CRNFPC & -31.0144 & \\
\hline CRCIWC & -30.7978 & \\
\hline CHMSWC & -30.7958 & \\
\hline CRWHVC & -30.2715 & \\
\hline CCMSWC & -29.7784 & \\
\hline CRYLWC & -29.6237 & \\
\hline
\end{tabular}


U. S. F. Tambunan et al.: Computational design of drug candidates for influenza A virus subtype H1N1 by inhibiting the viral neuraminidase-1 enzyme, Acta Pharm. 64 (2014) 157-172.

Table I. continued

\begin{tabular}{|c|c|c|}
\hline Ligand & $\Delta G\left(\mathrm{kcal} \mathrm{mol}^{-1}\right)$ & Structure \\
\hline CRHKIC & -29.6196 & \\
\hline CRHALC & -29.3549 & \\
\hline CNCSMC & -29.2081 & \\
\hline CHSMFC & -29.1800 & \\
\hline CDLHWC & -29.1791 & \\
\hline CRLEVC & -29.1223 & \\
\hline CDFCWC & -29.0683 & \\
\hline AD3BF2Da & -20.8098 & \\
\hline
\end{tabular}


U. S. F. Tambunan et al.: Computational design of drug candidates for influenza A virus subtype H1N1 by inhibiting the viral neuraminidase-1 enzyme, Acta Pharm. 64 (2014) 157-172.

Table I. continued

Ligand $\Delta \mathrm{G}\left(\mathrm{kcal} \mathrm{mol}^{-1}\right)$

a Standard

computed under thermodynamically equilibrium conditions. The value of $\Delta G$ binding described the binding strength of enzyme and ligand. Out of the 200 best ligands, which were repeated, 15 were obtained with the lowest Gibbs energy. The docking results are seen in Table I.

Among the fifteen ligands selected based on the lowest $\Delta G_{\text {binding value, several did }}$ not have the properties of carcinogens and mutagens. In the design process of the antiviral compound, ADMET (absorption, distribution, metabolism, excretion and toxicity) should be considered as an important step. Those properties were detected using the software ToxTree, Osiris Property Explore and FAF-drugs (23).

From the screening results using Toxtree software, it can be inferred that the fifteen ligands do not have mutagenic and carcinogenic properties. The screening results were obtained using the software Osiris Property Explore. It showed that ligands CRWHVC, CCMSWC and CRYLWC are mutagenic. CRWHVC ligand also has the potential to be tumorigenic, while the standard ligand oseltamivir possesses tumorigenic and mutagenic potential, which is less than that of the three ligands above. In addition to the four ligands, all ligands lack mutagenic and tumorigenic potential, irritation but have particularly effective reproductive values. Judging from the value of drug-likeness, similarities are observed within the properties of the existing drugs, because only two ligands have a good value compared to zanamivir, the standard ligand, namely CRMYPC and CRNFPC.

The main properties were screened by FAF-drugs including total H-bonds, tPSA (topological polar surface area) value, rotatable bonds, and $\log P(23)$. From the software FAF-drugs, CRMYPC and CRNFPC showed good bioavailability. However, CRMYPC had unfavorable bioavailability compared to CRNFPC. 
U. S. F. Tambunan et al.: Computational design of drug candidates for influenza A virus subtype H1N1 by inhibiting the viral neuraminidase-1 enzyme, Acta Pharm. 64 (2014) 157-172.
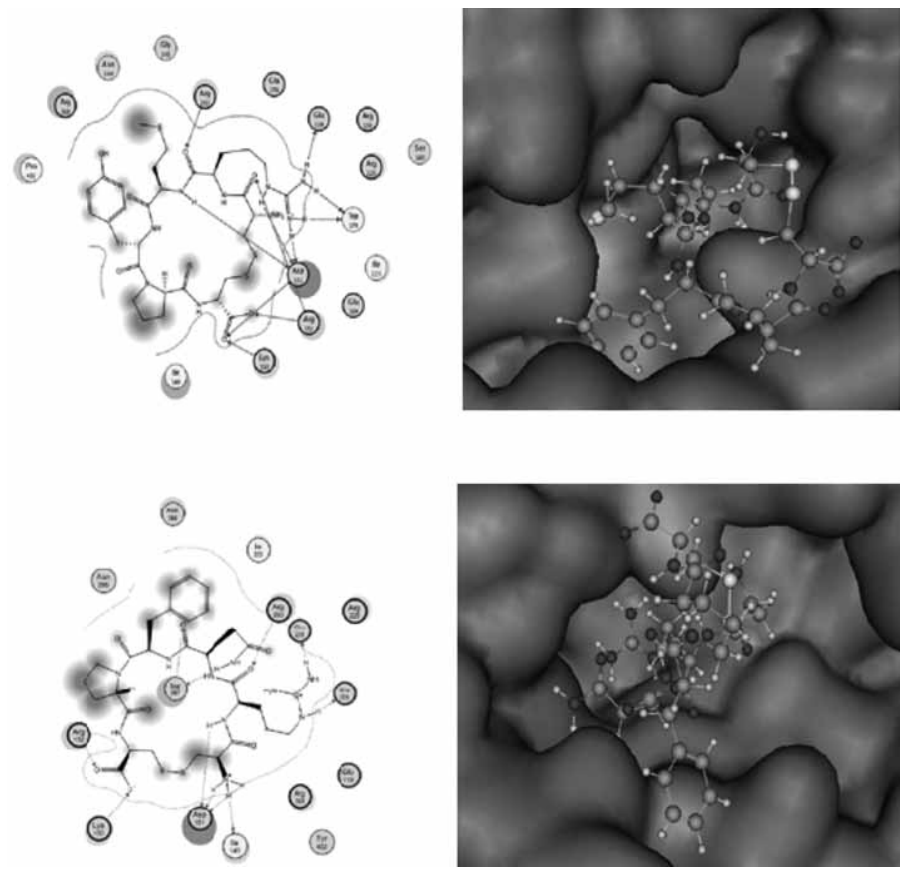

Fig. 3. a) The interaction of CRMYPC ligand with neuraminidase, b) the interaction of CRNFPC ligand with neuraminidase (MOE 2008.10).

CRMYPC and CRNFPC form fifteen and thirteen hydrogen bonds with the whole neuraminidase- 1 amino acid residues, respectively, while the standard ligands, namely AD3BF2D, NNY, oseltamivir and zanamivir have six, eight, three and eight hydrogen bonds, respectively. CRMYPC and CRNFPC have formed seven and eight hydrogen bonds with active site residue of neuraminidase-1, respectively, while AD3BF2D, NNY and zanamivir have three, three and four hydrogen bonds, respectively. Although the standards have much less hydrogen bonding than our ligands, they could still perform as drugs. We argue that stronger hydrogen bonding in our ligands will eventually produce a more robust ligand-receptor interaction. The results of $\Delta G_{\text {binding }}$ for both CRMYPC and CRNFPC were more negative than that of the standard ligands. Oseltamivir and other standard ligands have not formed hydrogen bonding with the active site residue of neuraminidase- 1 because of the mutation in the neuraminidase- 1 amino acid residues. This indicates that neuraminidase- 1 is resistant to oseltamivir.

Interaction between the ligand and CRNFPC and CRMYPC is seen in Figs. $3 a$ and $b$. CRMYPC and CRNFPC ligand conformations cover and fill the cavity of neuraminidase-1, so that these enzyme binding site ligands are proposed to act as competitive inhibitors. The potential binding site ligands bind to the enzyme and interfere with the catalytic activity of the enzyme. 
U. S. F. Tambunan et al.: Computational design of drug candidates for influenza A virus subtype H1N1 by inhibiting the viral neuraminidase-1 enzyme, Acta Pharm. 64 (2014) 157-172.
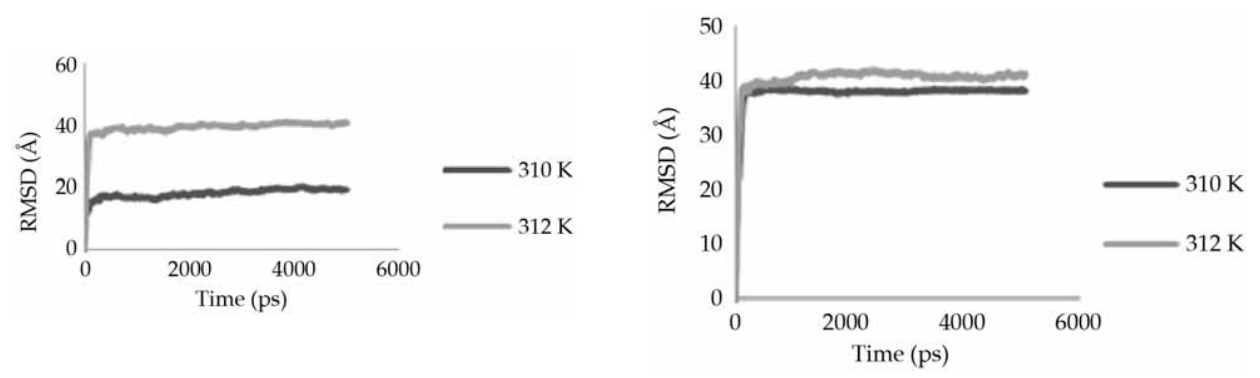

Fig. 4. RMSD vs. simulation time for: a) CRMYPC ligand, b) CRNFPC ligand.

CRMYPC interaction with neuraminidase- 1 shows some of the interactions between amino acid residues in the active site of neuraminidase- 1 with the ligand through hydrogen bonding. It happens, among others, with Asp151, Arg293 and Arg368 side chains that occur with arginine (amino acid code: R). Moreover, the interactions of residues Asp151 with cysteine (amino acid code: C) backbone could be observed as well.

CRNFPC ligand interacts with neuraminidase- 1 through different hydrogen bonds than ligand CRMYPC. Amino acid residues in the active site of neuraminidase- 1 that show hydrogen-bonding interaction are Asp151, Glu278 and Arg293 in the side chain of cysteine $(\mathrm{C})$ and arginine $(\mathrm{R})$.

Molecular dynamics was employed to study the effect of the presence of an explicit solvent system to explore the conformation of the receptor protein.

In a solution, protein is a dynamic molecule that does not have a single conformation. Conformational change of the enzyme-ligand complex can be seen from the full root mean square deviation (RMSD) vs. time simulation.

From Fig. 4, it is seen that the conformation of the ligand-enzyme complex at the beginning of the simulation (duration of $0-100 \mathrm{ps}$ ) is very volatile due to exponential climb of the energy function, and the simulation time is quite stable beyond $100 \mathrm{ps}$. The patterns in the graph that tend to be linear demonstrate this trend. Changes in the curve at the beginning of the simulation (0-100 ps) occur for both complexes, CRMYPC and CRNFPC, with considerable fluctuations. This indicates that the conformational changes of the structure of the ligand-enzyme complex are large enough during that time span. During the time of 100 ps, the simulation shows that the curve becomes linear. It suggests that, in this time span, the conformation of ligand-enzyme complex structure does not change significantly.

Increasing the temperature will result in increased movement of proteins. However, it can not be inferred whether the interactions between the protein and ligand become unstable. This is demonstrated by the insignificant increase in the RMSD value. The CRMYPC-enzyme complex undergoes an insignificant conformational change during the rising temperature. This ligand is slightly more reactive at temperatures of $312 \mathrm{~K}$ (fever body temperature) compared to $310 \mathrm{~K}$ temperature (normal body temperature), as can be seen from the tiny change in the value of RMSD. However, the CRNFPC-enzyme complex RMSD values at 300 and $312 \mathrm{~K}$ do not vary that much. 
U. S. F. Tambunan et al.: Computational design of drug candidates for influenza A virus subtype H1N1 by inhibiting the viral neuraminidase-1 enzyme, Acta Pharm. 64 (2014) 157-172.

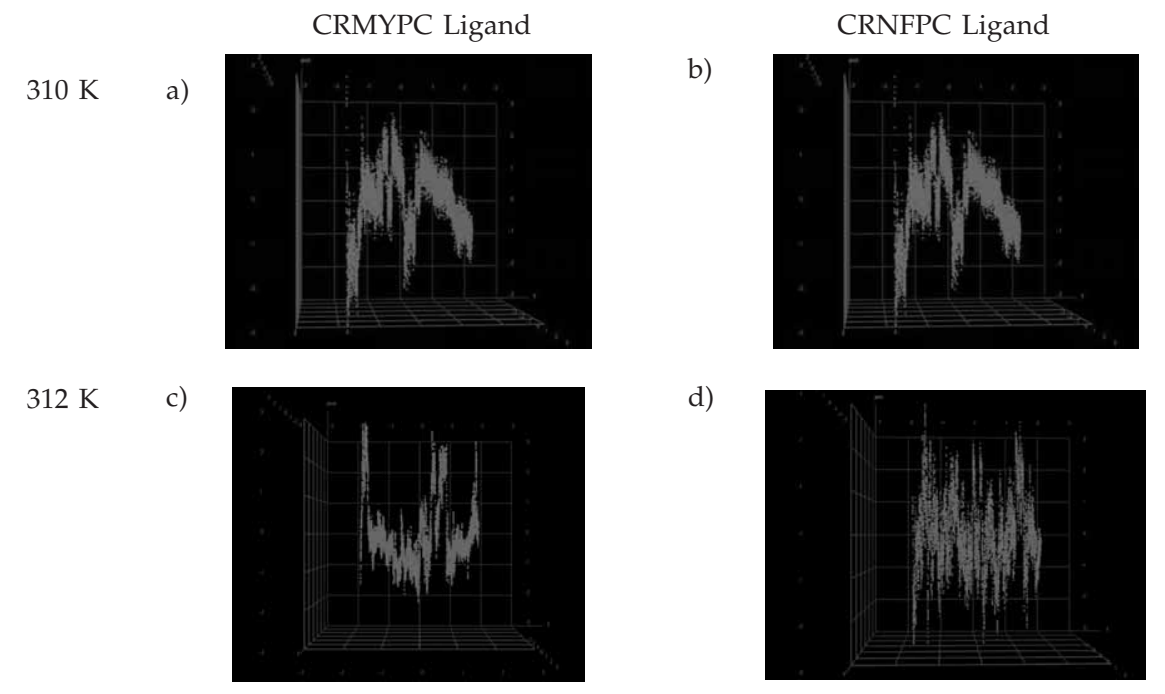

Fig. 5. The time-dependent distance graph for CRMYPC and CRNFPC ligands at 310 and $312 \mathrm{~K}$. Axis $x$ depicts the time function, while axis $y$ depicts the position function.

Differences in RMSD vs. timeplots that occur in CRMYPC-enzyme complex at temperatures of $310 \mathrm{~K}$ and $312 \mathrm{~K}$ seem noticeable compared to the curve in the CRNFPC-enzyme complex (Fig. 4). These changes can be attributed to the stability of the complex formation. CRMYPC sequences are composed of polar-polar-non-polar-polar-non-polar-polar residues, which are slightly more stable at temperatures below $310 \mathrm{~K}$. However, the CRNFPC sequences are composed of polar-polar-non-polar-polar-non-polar-polar residues. Hence, we argue that the curve differences will not affect the total stability of the complex due to the linear curves trend (Fig. 3a). Due to constant stability of the structure, the curve difference is totally negligible.

The stability of protein and ligand could be seen from the correlation plot between the total potential energy of the system during simulation. Decrease of the total potential energy system from three protein-ligand complex during simulation is an indication of a stable system. This can be seen from the amount of interactions that occur at the enzyme-binding site of both CRMYPC and CRNFPC.

The time-dependent distance graphs for CRMYPC and CRNFPC ligands at 310 and $312 \mathrm{~K}$ have shown that the distance fluctuations in the ligand-receptor complex are attributed to the Brownian motion (29) (Fig. 5).

The toxicity data of our designed ligands and the standards can be found in the supplementary material (available upon request). However, as we sum up the data into Table I, it can be seen that most of our designed ligands have no observable malignant effects. Hence, most of them are not in accord with Lipinski's rules. However, based upon our docking and dynamics results, these ligands could still be utilized as drug candidates. 
U. S. F. Tambunan et al.: Computational design of drug candidates for influenza A virus subtype H1N1 by inhibiting the viral neuraminidase-1 enzyme, Acta Pharm. 64 (2014) 157-172.

Table II. The Utilization of ADME-TOX softwares (Toxtree, Osiris, and FAF2) toward the ligands

\begin{tabular}{|c|c|c|c|}
\hline Ligand & Toxtree & Osiris Explorer & FAF2 Drug \\
\hline CRMYPC & - & - & \multirow{15}{*}{$\begin{array}{c}\text { Beyond } \\
\text { threshold } \\
\text { molecular } \\
\text { mass }\end{array}$} \\
\hline CWEVCC & - & - & \\
\hline CRNFPC & - & - & \\
\hline CRCIWC & - & - & \\
\hline CHMSWC & - & - & \\
\hline CRWHVC & - & $\begin{array}{l}\text { High mutagenicity, mild } \\
\text { tumorigenicity }\end{array}$ & \\
\hline CCMSWC & - & High mutagenicity & \\
\hline CRYLWC & - & High mutagenicity & \\
\hline CRHKIC & - & - & \\
\hline CRHALC & - & - & \\
\hline CNCSMC & - & - & \\
\hline CHSMFC & - & - & \\
\hline CDLHWC & - & - & \\
\hline CRLEVC & - & - & \\
\hline CDFCWC & - & - & \\
\hline $\mathrm{AD} \mathrm{BF} 2 \mathrm{D}^{\mathrm{a}}$ & $\begin{array}{l}\text { Positive for genotoxic } \\
\text { carcinogenicity }\end{array}$ & irritant & - \\
\hline NNYa & - & - & - \\
\hline Zanamivir $^{\mathrm{a}}$ & - & - & - \\
\hline Oseltamivir ${ }^{\mathrm{a}}$ & & Mild tumorigenic and mutagenic & - \\
\hline
\end{tabular}

a Standard

- No observable malignant effect.

\section{CONCLUSIONS}

Based on the simulation results of molecular docking and drug scan, there are two ligands that have good values of the residue target interaction and toxicological properties. These ligands are CRMYPC and CRNFPC. Both ligands have $\Delta G_{\text {binding values of }}$ -31.7402 and $31.0144 \mathrm{kcal} \mathrm{mol}^{-1}$, they are not mutagenic and carcinogenic and have good oral bioavailability.

Based on the evaluation of molecular dynamics simulations of the ligand in the hydrated state, CRMYPC ligand has hydrogen-bonding interactions with residues of the active site of neuraminidase- 1 . At the temperature of $310 \mathrm{~K}$, it formed a hydrogen bond with Arg368, Tyr402, Glu425, while at 312 K, it formed a hydrogen bond with Asp151. Differences in enzyme conformation during the simulation show the effect of temperature on enzyme activity. The results above indicate that the CRMYPC ligand can be used as neuraminidase-1 inhibitor candidate against the influenza virus A H1N1. 
U. S. F. Tambunan et al.: Computational design of drug candidates for influenza A virus subtype H1N1 by inhibiting the viral neuraminidase-1 enzyme, Acta Pharm. 64 (2014) 157-172.

In order to prove the efficacy of the ligand, wet laboratory experiments of in vitro and in vivo methods should be utilized. In this way, the drug candidate will be prepared for the clinical trial process.

Acknowledgements. - The authors express their gratitude to Hibah Pasca Sarjana DRPM UI Hibah PUPT BOPTN Ditjen Dikti No. 2788/H2.R12/HKP.05.00/2013. Thanks also go to Syarifuddin Idrus for the technical assistance, and Niken Widiyanti for the proof reading of this manuscript.

Acronyms, symbols, abbreviations. - Ala - alanine, Arg - arginine, Asn - asparagine, Asp - aspartic acid, Asx - asparagine or aspartic acid, $\Delta G_{\text {binding }}$ - binding energy, Calpha $(C \alpha)$ - asymetric carbon atom in the single amino acid, $\mathrm{C} \alpha-\mathrm{C}$ - covalent bond between carbon terminal and asymetric carbon atom in the single amino acid, Cys - cysteine, FASTA - FAST-All, Gal - D-galactose, Gln glutamine, Glu - glutamic acid, Glx - glutamine or glutamic acid, Gly - glycine, HBA - total amount of hydrogen atom acceptor, HBD - total amount of hydrogen atom donor, His - histidine, Ile - isoleucine, Leu - leucine, Lys - lysine, Met - methionine, MOE - molecular operating environment, N-C $\alpha$ covalent bond between nitrogen terminal and asymetric carbon atom in the single amino acid, NCBI - National Center for Biotechnology Information, LogP - partition coeficient, Phe - phenylalanine, Pro - proline, PDB - Protein Data Bank, RMS - Root Mean Square, RMSD - Root Mean Square Deviation, Ser - serine, Thr - threonine, Trp - tryptophan, Tyr - tyrosine, Val - valine.

\section{REFERENCES}

1. J. K. Taubenberger and D. M. Morens, 1918 Influenza: the mother of all pandemic, Emerg. Infect. Dis. 12 (2006) 15-22; DOI: 10.3201/eid1201.050979.

2. P. Auewarakul, O. Suptawiwat, A. Kongchanagul, C. Sangma, Y. Suzuki, K. Ungchusak, S. Louisirirotchanakul, H. Lerdsamran, P. Pooruk, A. Thitithanyanont, C. Pittayawonganon, C.-T. Guo, H. Hiramatsu, W. Jampangern, S. Chunsutthiwat and P. Puthavathana, An avian influenza H5N1 virus that binds to a human-type receptor, J. Virol. 81 (2007) 9950-9955; DOI: 10.1128/ JVI.00468-07.

3. I. V. Alymova, A. Portner, V. P. Mishin, J. A. McCullers, P. Freiden and G. L. Taylor, Receptor-binding specificity of the human parainfluenza virus type 1 hemagglutinin-neuraminidase glycoprotein, Glycobiology 22 (2012) 174-180; DOI: 10.1093/glycob/cwr112.

4. S.-Q. Wang, Q.-S. Du, R.-B. Huang, D.-W. Zhang and K.-C. Chou, Insights from investigating the interaction of oseltamivir (Tamiflu) with neuraminidase of the 2009 H1N1 swine flu virus, Biochem. Biophys. Res. Commun. 386 (2009) 432-436; DOI: 10.1016/j.bbrc.2009.06.016.

5. H. T. Nguyen, T. G. Sheu, V. P. Mishin, A. I. Klimov and L. V Gubareva, Assessment of pandemic and seasonal influenza A (H1N1) virus susceptibility to neuraminidase inhibitors in three enzyme activity inhibition assays, Antimicrob. Agents Chemother. 54 (2010) 3671-3677; DOI: 10.1128/ AAC.00581-10.

6. N. J. Dharan, L. V. Gubareva, J. J. Meyer, M. Okomo-Adhiambo, R. C. McClinton, S. A. Marshall, K. St George, S. Epperson, L. Brammer, A. I. Klimov, J. S. Bresee and A. M. Fry, Infections with oseltamivir-resistant influenza A(H1N1) virus in the United States, JAMA 301 (2009) 1034-1041; DOI: 10.1001/jama.2009.294.

7. T. Kolomin, M. Shadrina, P. Slominsky, S. Limborska and N. Myasoedov, A new generation of drugs: Synthetic peptides based on natural regulatory peptides, Neurosci. Med. 4 (2013) 223-252; DOI: $10.4326 / \mathrm{nm} .2013 .44035$.

8. C. D. Fjell, J. A. Hiss, R. E. W. Hancock and G. Schneider, Designing antimicrobial peptides: form follows function, Nat. Rev. Drug Discov. 11 (2012) 37-51; DOI: 10.1038/nrd3591. 
U. S. F. Tambunan et al.: Computational design of drug candidates for influenza A virus subtype H1N1 by inhibiting the viral neuraminidase-1 enzyme, Acta Pharm. 64 (2014) 157-172.

9. S. Riedl, D. Zweytick and K. Lohner, Membrane-active host defense peptides - challenges and perspectives for the development of novel anticancer drugs, Chem. Phys. Lipids 164 (2011) 766-781; DOI: 10.1016/j.chemphyslip.2011.09.004.

10. P. Vlieghe, V. Lisowski, J. Martinez and M. Khrestchatisky, Synthetic therapeutic peptides: science and market, Drug. Disc. Today 15 (2010) 40-56; DOI: 10.1016/j.drudis.2009.10.009.

11. R. Benigni and C. Bossa, Structure alerts for carcinogenicity, and the Salmonella assay system: a novel insight through the chemical relational databases technology, Mutat. Res. 659 (2008) 248-261; DOI: 10.1016/j.mrrev.2008.05.003.

12. U. S. F. Tambunan, N. Amri and A. A. Parikesit, In silico design of cyclic peptides as influenza virus, a subtype H1N1 neuraminidase inhibitor, African J. Biotechnol. 11 (2012) 11474-11491; DOI: 10.5897/ AJB11.4094.

13. C. A. Lipinski, F. Lombardo, B. W. Dominy and P. J. Feeney, Experimental and computational approaches to estimate solubility and permeability in drug discovery and development settings, Adv. Drug Deliv. Rev. 46 (2001) 3-26.

14. S.-L. Du, H. Pan, W.-Y. Lu, J. Wang, J. Wu and J.-Y. Wang, Cyclic Arg-Gly-Asp peptide-labeled liposomes for targeting drug therapy of hepatic fibrosis in rats, J. Pharmacol. Exp. Ther. 322 (2007) 560-568; DOI: 10.1124/jpet.107.122481.

15. T. R. White, C. M. Renzelman, A. C. Rand, T. Rezai, C. M. McEwen, V. M. Gelev, R. A. Turner, R. G. Linington, S. S. F. Leung, A. S. Kalgutkar, J. N. Bauman, Y. Zhang, S. Liras, D. A. Price, A. M. Mathiowetz, M. P. Jacobson and R. S. Lokey, On-resin N-methylation of cyclic peptides for discovery of orally bioavailable scaffolds, Nat. Chem. Biol. 7 (2011) 810-817; DOI: 10.1038/nchembio. 664.

16. M. Brvar, A. Perdih, M. Renko, G. Anderluh, D. Turk and T. Solmajer, Structure-based discovery of substituted 4,5'-bithiazoles as novel DNA gyrase inhibitors, J. Med. Chem. 55 (2012) 6413-6426; DOI: $10.1021 /$ jm300395d.

17. C. Liao, M. Sitzmann, A. Pugliese and M. C. Nicklaus, Software and resources for computational medicinal chemistry, Future Med. Chem. 3 (2011) 1057-1085; DOI: 10.4155/fmc.11.63.

18. D. L. Wheeler, T. Barrett, D. A. Benson, S. H. Bryant, K. Canese, V. Chetvernin, D. M. Church, M. DiCuccio, R. Edgar, S. Federhen, L. Y. Geer, Y. Kapustin, O. Khovayko, D. Landsman, D. J. Lipman, T. L. Madden, D. R. Maglott, J. Ostell, K. D. Pruitt, G. D. Schuler, L. M. Schriml, E. Sequeira, S. T. Sherry, K. Sirotkin, A. Souvorov, G. Starchenko, T. O. Suzek, R. Tatusov, T. A. Tatusova, L. Wagner and E. Yaschenko, Database resources of the National Center for Biotechnology Information, Nucl. Acids Res. 35 (2007) D5-D12; DOI: 10.1093/nar/gkl1031.

19. U. S. F. Tambunan, R. Harganingtyas and A. A. Parikesit, In silico modification of $(1 R, 2 R, 3 R$, $5 S)-(-)$-isopinocampheylamine as inhibitors of M2 proton channel in Influenza A virus subtype H1N1, using the molecular docking approach, Trends Bioinform. 5 (2012) 25-46; DOI: 10.3923/ tb.2012.25.46.

20. U. S. F. Tambunan, N. Bramantya and A. A. Parikesit, In silico modification of suberoylanilide hydroxamic acid (SAHA) as potential inhibitor for class II histone deacetylase (HDAC), BMC Bioinformatics 12 (Suppl. 13) (2011) S23-S38; DOI:10.1186/1471-2105-12-S13-S23.

21. R. Benigni and C. Bossa, Structure alerts for carcinogenicity, and the Salmonella assay system: a novel insight through the chemical relational databases technology, Mutat. Res. 659 (2008) 248-261; DOI: $10.1016 /$ j.mrrev.2008.05.003.

22. T. Sander, J. Freyss, M. von Korff, J. R. Reich and C. Rufener, OSIRIS, an entirely in-house developed drug discovery informatics system, J. Chem. Inf. Model. 49 (2009) 232-246; DOI: 10.1021/ ci800305f.

23. D. Lagorce, O. Sperandio, H. Galons, M. A. Miteva and B. O. Villoutreix, FAF-drugs2: free ADME/ tox filtering tool to assist drug discovery and chemical biology projects, BMC Bioinformatics 9 (2008) 396-405; DOI: 10.1186/1471-2105-9-396. 
U. S. F. Tambunan et al.: Computational design of drug candidates for influenza A virus subtype H1N1 by inhibiting the viral neuraminidase-1 enzyme, Acta Pharm. 64 (2014) 157-172.

24. A. D. MacKerell, N. Banavali and N. Foloppe, Development and current status of the CHARMM force field for nucleic acids, Biopolymers 56 (2000) 257-265; DOI: 10.1002/1097-0282 (2000)56:4< 257::AID-BIP10029>3.0.CO;2-W.

25. Y. Shen, M. K. Gilson and B. Tidor, Charge optimization theory for induced-fit ligands, J. Chem. Theory Comput. 8 (2012) 4580-4592; DOI: 10.1021/ct200931c.

26. J. R. Williams, A. L. Khandoga, P. Goyal, J. I. Fells, D. H. Perygin, W. Siess, A. L. Parrill, G. Tigyi and Y. Fujiwara, Unique ligand selectivity of the GPR92/LPA5 lysophosphatidate receptor indicates role in human platelet activation, J. Biol. Chem. 284 (2009) 17304-17319; DOI: 10.1074/jbc. M109.003194.

27. G. L. Warren, C. W. Andrews, A.-M. Capelli, B. Clarke, J. LaLonde, M. H. Lambert, M. Lindvall, N. Nevins, S. F. Semus, S. Senger, G. Tedesco, I. D. Wall, J. M. Woolven, C. E. Peishoff and M. S. Head, A critical assessment of docking programs and scoring functions, J. Med. Chem.49 (2006) 5912-5931; DOI: 10.1021/jm050362n.

28. Y. Modis, S. Ogata, D. Clements and S. C. Harrison, A ligand-binding pocket in the dengue virus envelope glycoprotein, Proc. Natl. Acad. Sci. USA 100 (2003) 6986-6991; DOI: 10.1073/pnas. 0832193100 .

29. M. Takano, T. P. Terada and M. Sasai, Unidirectional Brownian motion observed in an in silico single molecule experiment of an actomyosin motor, Proc. Natl. Acad. Sci. USA 107 (2010) 77697774; DOI: 10.1073/pnas.0911830107.

30. S. Rogers, R. Wells and M. Rechsteiner, Amino acid sequences common to rapidly degraded proteins: the PEST hypothesis, Science 234 (1986) 364-368, DOI: 10.1126/science.2876518. 